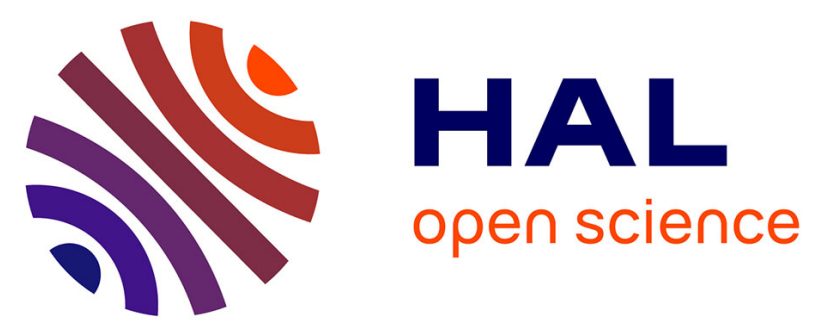

\title{
Particle Filter with Hybrid Importance Function for Joint Symbol Detection and Phase Tracking
}

\author{
François Septier, Yves Delignon, Atika Rivenq, Christelle Garnier
}

\section{To cite this version:}

François Septier, Yves Delignon, Atika Rivenq, Christelle Garnier. Particle Filter with Hybrid Importance Function for Joint Symbol Detection and Phase Tracking. IEEE 7th Workshop on Signal Processing Advances in Wireless Communications, Jul 2006, Cannes, France. pp.1-5, 10.1109/SPAWC.2006.346358 . hal-00685815

\section{HAL Id: hal-00685815 https://hal-imt.archives-ouvertes.fr/hal-00685815}

Submitted on 6 Apr 2012

HAL is a multi-disciplinary open access archive for the deposit and dissemination of scientific research documents, whether they are published or not. The documents may come from teaching and research institutions in France or abroad, or from public or private research centers.
L'archive ouverte pluridisciplinaire HAL, est destinée au dépôt et à la diffusion de documents scientifiques de niveau recherche, publiés ou non, émanant des établissements d'enseignement et de recherche français ou étrangers, des laboratoires publics ou privés. 


\title{
PARTICLE FILTER WITH HYBRID IMPORTANCE FUNCTION FOR JOINT SYMBOL DETECTION AND PHASE TRACKING
}

\author{
François Septier, Yves Delignon*, Atika Menhaj-Rivenq, Christelle Garnier* \\ IEMN-DOAE UMR 8520 \\ U.V.H.C. Le Mont Houy \\ 59313 Valenciennes Cedex 9 - FRANCE \\ *GET/INT - IEMN UMR 8520 \\ TELECOM Lille 1 \\ 59658 Villeneuve d'Ascq - FRANCE
}

\begin{abstract}
In digital communication, phase distortions introduced by local oscillators is one of the major concerns in designing low-cost high frequency wireless communication systems. Hence, for phase tracking and symbol detection, we propose an original algorithm which performs both phase tracking and symbol detection by a particle filtering based on the hybrid importance function. Performances of this algorithm are analyzed and compared to phaselocked loop performances in terms of bit error rate and mean square error. Asymptotic posterior Cramér-Rao bound is also derived to show performances of the optimum receiver.
\end{abstract}

Keywords : Phase tracking, Particle filter, Hybrid importance function, Decision feedback loop, Asymptotic posterior CramérRao bound.

\section{INTRODUCTION}

Synchronization is an important practical problem in communication systems. Indeed, non perfect local oscillators used in the receiver and the transmitter seriously damage the baseband received signal. These distortions correspond to the constant shift between the frequency clocks of the transmitter and the receiver and to the random jitter in frequency, known as phase noise, coming from electronic components. Moreover, the phase noise effect is much more significant when the carrier frequency of the digital communication system is large $[1,2]$ so that it has to be seriously fought against when designing future $40 \mathrm{GHz}$ or $60 \mathrm{GHz}$ communication systems.

The induced time variation of the frequency carrier is estimated and eliminated by a phase tracking device among which the phase-locked loop (PLL) and its digital version (DPLL) are the most commonly used devices.

Recent developments in signal processing and digital communications have shown that it is possible to avoid such approximations by using particle filters. Many wireless communication problems have been solved with success in that way [3] including detection in fast fading channels [4] and multiuser detection in fading code division multiple access (CDMA) channels $[5]$.

In [6], phase noise tracking performance with a prior importance function based particle filter has been examined in a bandlimited additive gaussian channel distortions and compared to phase-locked loop in term of Asymptotic Mean Square Error. In this approach, only the phase tracking problem has been considered.
In this paper, we focus our attention on the joint estimation of phase distortions and transmitted symbols. We propose a particle filter based on the hybrid importance function (PF-SDPT) introduced by Huang and Djuric [7]. Let us note that in [8], authors have proposed an algorithm which combines particle filtering and extended kalman filtering for this joint estimation. However, this approach does not take into account the linear drift and requires the linearization of the dynamic state space model which limits its performance especially when the phase noise rate increases.

The paper is organised as follows :

In section 2, the digital bandlimited communication system and the phase distortion model are described. Section 3 is devoted to particle filtering. We describe the proposed particle filter algorithm for joint phase tracking and symbol detection. Results are given in section 4 . The proposed algorithm is compared to the decision feedback loop (DFL) and particle filter algorithm (PF-PT) proposed by [6] in terms of bit error rate (BER) and mean square error (MSE) of phase estimation. We also derive the asymptotic posterior Cramér-Rao bound (PCRB) which corresponds to the lowest achievable MSE in the estimation of phase distortions.

\section{SYSTEM MODEL}

We consider the baseband communication system in a bandlimited gaussian channel shown in Fig. 1. Phase distortions at the receiver result from two phenomena. One is the constant shift between the frequency clocks of the transmitter and the receiver. The other is the random jitter introduced by the thermal noise of electronic components, known as phase noise. The constant drift and the phase noise are modeled by a multiplicative term $p(t)$ after the pulse shape filtering. In the case of an AWGN channel, $p(t)$ takes into account the phase noise produced by both the transmitter and the receiver.

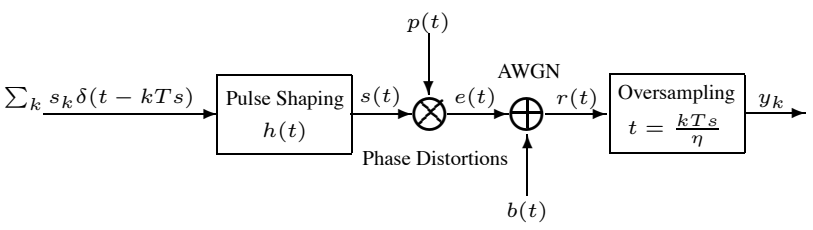

Fig. 1. Baseband system model. 
In a baseband complex equivalent form, the carrier delivered by the noisy oscillator can be modeled as :

$$
p(t)=\exp (j \theta(t))
$$

where the phase distortion $\theta(t)$ is a Brownian process with a linear drift [1]. Its power spectral density has a Lorentzian shape controlled by the parameter $\beta$ representing the two-sided $3 \mathrm{~dB}$ bandwidth. This model produces a $1 / f^{2}$ type noise power behavior that agrees with experimental measurements carried out on real RF oscillators. The phase noise rate is characterized by the bandwidth $\beta$ normalized with respect to the symbol rate $1 / T s$, namely by the parameter $\beta T s$.

At the sampling rate of the receiver $\eta / T s$, the discrete form of the phase distortion is :

$$
\theta_{k}=\theta_{k-1}+\varepsilon+v_{k}
$$

where $\varepsilon$ is the unknown constant drift between the transmitter and the receiver and $v_{k}$ is a zero mean gaussian variable with variance $\sigma_{v}^{2}=2 \pi \beta T s / \eta$.

The baseband transmitter delivers i.i.d symbols, which belong to an alphabet $A$, going through a pulse shaping filter. At the receiver, the maximum likelihood detection can be carried out either on the sampled signal following the matched filter, or directly from the oversampled received signal. In the first case, the Nyquist criterion for bandlimited transmission is not fulfilled, since phase noise causes additive and multiplicative distortions that are statistically intractable [9]. As a consequence, particle filter implementation after the matched filter is inappropriate.

Thereby, we focus on the maximum likelihood detection performed on the received signal which is oversampled by a factor $\eta$. In order to avoid Intersymbol Interference (ISI) at each sampling point, a multi-Nyquist pulse is used at the transmitter $[10,11]$.

\subsection{Dynamic state space model}

At the receiver side, the two unknown states are the transmitted symbols and the phase distortions. The nonlinear state space model can then be written as follows :

$$
\left\{\begin{array}{l}
\theta_{k}=\theta_{k-1}+\varepsilon+v_{k}, \\
y_{k}=\exp \left(j \theta_{k}\right) s_{\left\lfloor\frac{k}{\eta}\right\rfloor}+B_{k}
\end{array} \quad \varepsilon:\right. \text { unknown constant }
$$

where $B_{k} \sim N C\left(0, \sigma_{B}^{2}\right)$ is a circular zero mean gaussian white noise with power $\sigma_{B}^{2}$ and $v_{k} \sim N\left(0, \sigma_{v}^{2}\right)$ is a zero mean gaussian white noise with variance $\sigma_{v}^{2}$. The operator $\lfloor X\rfloor$ represents the largest integer lower than or equal to $X$. The system state is a three dimensional vector $\left(\varepsilon, s_{\left\lfloor\frac{k}{\eta}\right\rfloor}, \theta_{k}\right)$ where the phase varies at each sampling time, the symbols vary at each symbol time and $\varepsilon$ is constant during all the transmission and independent of both $v_{k}$ and the transmitted symbols. Moreover, the oversampling is at the origin of the following relation :

$$
\forall k \in\{1+i \eta, \ldots,(1+i) \eta-1 \mid i \in \mathbb{Z}\}, s_{\left\lfloor\frac{k}{\eta}\right\rfloor}=s_{\left\lfloor\frac{k-1}{\eta}\right\rfloor}
$$

\section{PARTICLE FILTER}

\subsection{Introduction}

The maximum a posteriori (MAP) estimation of the state from the measurement is obtained in the framework of the Bayesian theory which has been mainly investigated in Kalman filtering [12]. The Kalman filter is optimal when state and measurement equations are linear and noises are independent, additive and gaussian. When these assumptions are not fulfilled, various approximation methods have been developed among which the extended Kalman filter (EKF) is the most commonly used technique [12].

Since the nineties, particle filters have become a powerful methodology to cope with non-linear and non gaussian problems [13] and an important alternative to the EKF. The advantage lies in an approximation of the distribution of interest by discrete random measures, which does not involve any linearization.

Based on sequential importance sampling, particle filtering is an extension of the sequential Monte Carlo methodology [14]. It consists in recursively computing the required posterior density function $p\left(x_{0: k} \mid y_{0: k}\right)$ by approximating by a set of $N$ random samples with associated weights, denoted by $\left\{x_{0: k}^{(m)}, w_{k}^{(m)}\right\}_{m=1 . . N}$ :

$$
\widehat{p}\left(x_{0: k} \mid y_{0: k}\right)=\sum_{j=1}^{N} \delta\left(x_{0: k}-x_{0: k}^{(j)}\right) \widetilde{w}_{k}^{(j)}
$$

where $x_{k}^{(j)}$ is drawn from the importance function $\pi\left(x_{k} \mid x_{0: k-1}^{(m)}, y_{0: k}\right)$,

$\delta($.$) is the Dirac delta function and$

$\widetilde{w}_{k}^{(j)}=w_{k}^{(j)} / \sum_{m=1}^{N} w_{k}^{(m)}$ is the normalized importance weight associated with the $j$-th particle.

The weights $w_{k}^{(m)}$ are updated according the concept of importance sampling :

$$
w_{k}^{(m)} \propto \frac{p\left(y_{k} \mid x_{0: k}^{(m)}\right) p\left(x_{k}^{(m)} \mid x_{0: k-1}^{(m)}\right)}{\pi\left(x_{k}^{(m)} \mid x_{0: k-1}^{(m)}, y_{0: k}\right)} w_{k-1}^{(m)}
$$

In order to overcome the degeneracy problems of particle filtering after few iterations $[14,15]$, we integrate in the algorithm a resampling step as in [16].

Performances of particle filter mainly depend on the choice of the importance function. The most common choices are the prior density function and the optimal density function. The first one is the most widely used [17], which is largely due to the implementation simplicity. However, this distribution does not incorporate any information contained in the most recent observation and, therefore, may be inefficient and especially sensitive to outliers. The optimal density based particle filter has been introduced in [18]. In this paper, Zaritskii has shown that it minimizes the variance of the importance weights $\widetilde{w}_{k}^{(m)}$ conditional upon the particle trajectories $x_{0: k}^{(m)}$ and the observations $y_{0: k}$. When dealing with a state process which can be divided into two independent parts, Huang and Djuric have proposed the hybrid importance function [7] which combines the prior and the optimal density.

\subsection{Particle filtering for phase tracking and symbol detection}

In this paragraph, we develop particle filter based on the hybrid (PF-SDPT) for joint phase distortion estimation and symbol detection.

The design of the particle filter requires both a simulation method for the generation of particles and the formula for updating importance weights. Since the state vector we want to estimate $x_{k}=\left(\varepsilon, \theta_{k}, s_{\left\lfloor\frac{k}{n}\right\rfloor}\right)$ includes three processes among which 
only the phase $\theta_{k}$ and the transmitted symbol $s_{\left\lfloor\frac{k}{\eta}\right\rfloor}$ are time varying, the particle generation is carried out at steps 0 and 1 . The step 0 consists in drawing each particle coordinate $\left\{\varepsilon^{(m)}\right\}_{m=1 . . N}$ according to the uniform law on a predefined range and does not depend on the importance function unlike steps 1 and 2 .

In our context, this hybrid importance function can be written as :

$$
\begin{array}{r}
\pi\left(s_{\left\lfloor\frac{k}{\eta}\right\rfloor}, \theta_{k} \mid s_{0:\left\lfloor\frac{k-1}{\eta}\right\rfloor}^{(m)}, \theta_{0: k-1}^{(m)}, y_{0: k}\right)=p\left(\theta_{k} \mid \theta_{k-1}^{(m)}\right) \\
\quad \times p\left(s_{\left\lfloor\frac{k}{\eta}\right\rfloor} \mid s_{0:\left\lfloor\frac{k-1}{\eta}\right\rfloor}^{(m)}, \theta_{0: k}^{(m)}, y_{0: k}\right)
\end{array}
$$

where

$$
p\left(\theta_{k} \mid \theta_{k-1}^{(m)}\right)=N\left(\theta_{k} ; \theta_{k-1}^{(m)}+\varepsilon^{(m)}, \sigma_{v}^{2}\right)
$$

and,

$p\left(s_{\left\lfloor\frac{k}{\eta}\right\rfloor} \mid s_{0:\left\lfloor\frac{k-1}{\eta}\right\rfloor}^{(m)}, \theta_{0: k}^{(m)}, y_{0: k}\right)=\frac{p\left(y_{k} \mid s_{\left\lfloor\frac{k}{\eta}\right\rfloor}, \theta_{k}^{(m)}\right) p\left(s_{\left\lfloor\frac{k}{\eta}\right\rfloor} \mid s_{0:\left\lfloor\frac{k-1}{\eta}\right\rfloor}^{(m)}\right)}{\sum_{s_{\left\lfloor\frac{k}{\eta}\right\rfloor} \in A} p\left(y_{k} \mid \theta_{k}^{(m)}, s_{\left\lfloor\frac{k}{\eta}\right\rfloor}\right)}$

This last expression can be simplified when $k$ is a multiple of $\eta$ by :

$$
p\left(s_{\left\lfloor\frac{k}{\eta}\right\rfloor} \mid s_{0:\left\lfloor\frac{k-1}{\eta}\right\rfloor}, \theta_{0: k}, y_{0: k}\right)=\frac{p\left(y_{k} \mid s_{\left\lfloor\frac{k}{\eta}\right\rfloor}, \theta_{k}\right)}{\operatorname{card}(A) p\left(y_{k} \mid \theta_{k}, s_{\left\lfloor\frac{k-1}{\eta}\right\rfloor}\right)}
$$

where

$$
p\left(y_{k} \mid \theta_{k}, s_{\left\lfloor\frac{k}{\eta}\right\rfloor}\right)=N C\left(y_{k} ; s_{\left\lfloor\frac{k}{\eta}\right\rfloor} e^{j \theta_{k}}, \sigma_{B}^{2}\right)
$$

Otherwise, (9) takes the form :

$$
p\left(s_{\left\lfloor\frac{k}{\eta}\right\rfloor} \mid s_{0:\left\lfloor\frac{k-1}{\eta}\right\rfloor}, \theta_{0: k}, y_{0: k}\right)= \begin{cases}1 & \text { if } s_{\left\lfloor\frac{k}{\eta}\right\rfloor}=s_{\left\lfloor\frac{k-1}{\eta}\right\rfloor} \\ 0 & \text { otherwise }\end{cases}
$$

The PF-SDPT does not take into account the received signal for sampling $\theta_{k}$. Indeed, we draw $N$ samples $\theta_{k}^{(m)}$ from the prior probability density function $p\left(\theta_{k} \mid \theta_{0: k-1}^{(m)}\right)$ given by (8). In this context, the update of the weights can be computed using (11) as :

$$
w_{k}^{(m)} \propto \frac{1}{\operatorname{card}(A)} \sum_{s_{\left\lfloor\frac{k}{\eta}\right\rfloor} \in A} p\left(y_{k} \mid s_{\left\lfloor\frac{k}{\eta}\right\rfloor}, \theta_{k}^{(m)}\right) w_{k-1}^{(m)}
$$

, if $k$ is a multiple of $\eta$.

$$
w_{k}^{(m)} \propto p\left(y_{k} \mid s_{\left\lfloor\frac{k}{\eta}\right\rfloor}=s_{\left\lfloor\frac{k-1}{\eta}\right\rfloor}, \theta_{k}^{(m)}\right) w_{k-1}^{(m)}
$$

, otherwise.

Every element required in the implementation of the particle filtering algorithm have been identified. The miniminum mean square error (MMSE) estimate of $\theta_{k}$ can be easily calculated according to :

$$
\widehat{\theta}_{k}^{m m s e}=\sum_{j=1}^{M} \theta_{k}^{(j)} \widetilde{w}_{k}^{(j)}
$$

and the maximum a posteriori (MAP) estimate of the data performed at the end of each symbol (i.e. $k+1$ multiple of $\eta$ ) is

$\widehat{s}_{\left\lfloor\frac{k}{\eta}\right\rfloor}^{\operatorname{map}}=\operatorname{argmax}_{\left\lfloor\frac{k}{\eta}\right\rfloor}\left\{\sum_{j=1}^{M} \widetilde{w}_{k}^{(j)} \delta\left(\left(\theta_{k}, s_{\left\lfloor\frac{k}{\eta}\right\rfloor}\right)-\left(\theta_{k}, s_{\left\lfloor\frac{k}{\eta}\right\rfloor}\right)^{(j)}\right)\right\}$

The PF-SDPT algorithm is summed up in Table 1.
Table 1. PF-SDPT algorithm

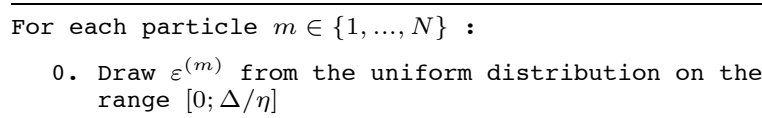

1. (a) Draw particles using (8) :

$$
\theta_{k}^{(m)} \sim p\left(\theta_{k} \mid \theta_{k-1}^{(m)}\right)
$$

(b) Draw particles :

$$
s_{\left\lfloor\frac{k}{\eta}\right\rfloor}^{(m)} \sim p\left(s_{\left\lfloor\frac{k}{\eta}\right\rfloor} \mid s_{0:\left\lfloor\frac{k-1}{\eta}\right\rfloor}^{(m)}, \theta_{0: k}^{(m)}, y_{0: k}\right)
$$

If $k$ multiple of $\eta$, use (10) and (11) else, use (12)

2. Evaluate the importance weights :

$$
w_{k}^{(m)} \propto p\left(y_{k} \mid \theta_{k}^{(m)}, s_{\left\lfloor\frac{k-1}{\eta}\right\rfloor}^{(m)}\right) w_{k-1}^{(m)}
$$

If $k$ multiple of $\eta$, use (13) else, use (14).

3. Normalize the importance weights : $\widetilde{w}_{k}^{(j)}=1 / \sum_{m=1}^{N} w_{k}^{(m)}$

4. Resampling step if $N_{\text {eff }}<N / 2$

\section{RESULTS}

The performance of the proposed particle filtering algorithm using hybrid importance function is studied for a binary phase shift keying (BPSK) modulation. We have randomly generated 2,000 signal bursts of duration $T=500 \mathrm{Ts}$ (i.e., 1,000,000 bits). The linear drift $\varepsilon$ is set at $0.5 / \eta$ which corresponds to a very bad synchronization. The particle filter algorithms have been implemented with 600 particles.

Let us note that the DFL and the PF-PT described in [6] are only phase tracking algorithms. Indeed, the maximum likelihood detection is carried out on the $\eta$ available samples of the received signal after phase distortion correction. In the proposed PF-SDPT, phase distortions and symbols are jointly estimated according to respectively MMSE and maximum a posteriori criteria.

Figure 2 shows the performance in term of BER achieved for different phase noise rates and with an oversampling factor $\eta=4$. It can be seen that the proposed PF-SDPT algorithm outperforms the DFL and the PF-PT whatever the signal-to-noise ratio (SNR). However smaller the SNR is, closer are the curves. The Mean Square Error (MSE) of the phase distortion estimates depicted in Figure 3 is obtained by :

$$
M S E=E\left[\left(\left(\theta_{k}-\widehat{\theta}_{k}\right)[2 \pi]\right)^{2}\right]
$$

where $\widehat{\theta}_{k}$ is the phase distortion estimate at time $k$. This MSE confirms that estimation is achieved by PF algorithms with a better accuracy. We can denote that MSE is always weaker with the PF-SDPT than with other algorithms. We can also remark in Figure 3 that the PF-SDPT is more robust when the phase noise rate increases. At $20 \mathrm{~dB}$, MSE of PF-SDPT and PF-PT are close to the optimum (asymptotic PCRB analytically derived in Appendix A) up to respectively $\beta T s=0.05$ and $\beta T s=0.04$. When the phase noise rate increases, the MSE increases rapidly due to the 
symbol error detection. Even if the MSE performances of the two $\mathrm{PF}$ algorithms are very close at high SNR and for a low phase noise rate, symbol detection by PF-SDPT is nevertheless better achieved than by PF-PT (Fig. 2). Since the PF-PT corrects the phase before the symbol detection, its performance in term of BER is related directly to the quality of the phase distortion estimate. On the other hand for the PF-SDPT, the symbol detection is based on the MAP criterion and performed jointly with the phase tracking, which improves to him more robustness and thus a greater effectiveness in detection.

Finally, we study the influence of the oversampling factor. Figure 4 represents the BER versus phase noise rate for two different values of $\eta$ and with a SNR fixed at $10 \mathrm{~dB}$. The figure shows that with $\eta=2$, the BER curves for all the algorithms degrade faster but they are still efficient for a low phase noise rate. The results of the proposed approaches are still better than the PFPT and the DFL results.

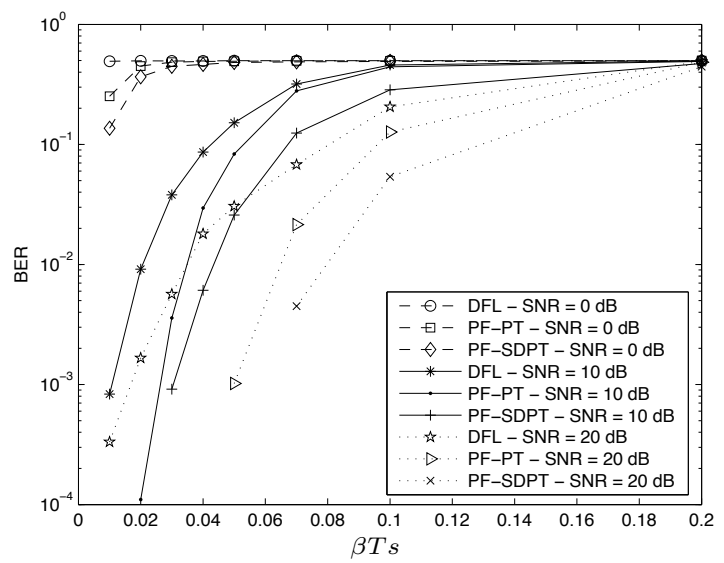

Fig. 2. BER of the algorithms versus phase noise rate $\beta T s$ with three different SNR and $\eta=4$.

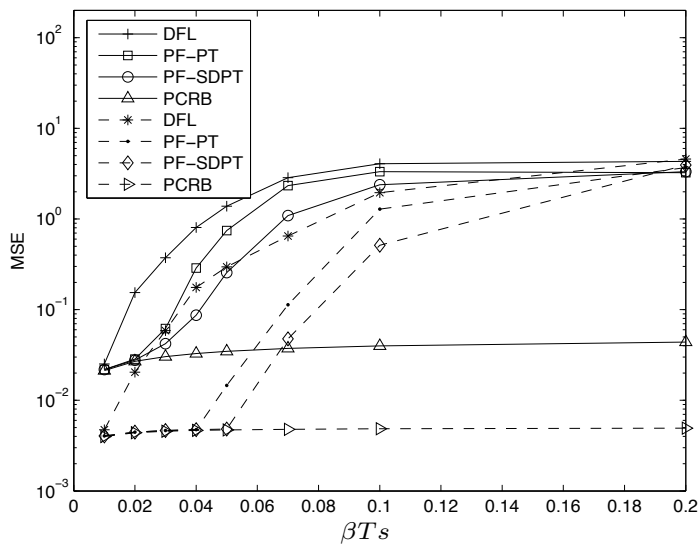

Fig. 3. MSE of the algorithms versus phase noise rate $\beta T s$ with $\mathrm{SNR}=10 \mathrm{~dB}$ (solid lines) and $\mathrm{SNR}=20$ (dashed lines).

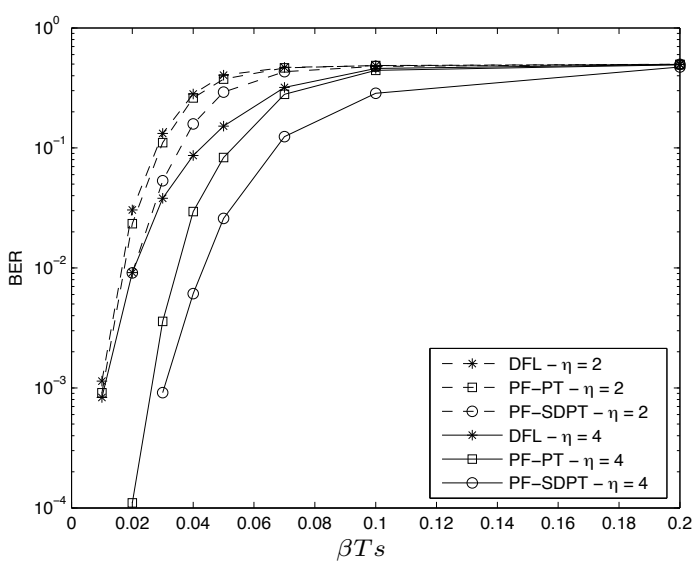

Fig. 4. BER of the algorithms versus phase noise rate $\beta T s$ with two different oversampling factors with $\mathrm{SNR}=10 \mathrm{~dB}$.

\section{CONCLUSION}

In this paper, we have considered the problem of phase synchronization in digital communications. To solve this problem, we have proposed a particle filter based on the hybrid importance function. The main originality of this work is the joint estimation of phase distortions and transmitted symbols by particle filtering based on the hybrid importance function. This one takes advantages of both the posterior and the prior importance function. Moreover, the algorithms are implemented in the case of multiple samples per symbol, which provides implicit diversity and allows a better phase tracking. Numerical simulations demonstrate the effectiveness of the proposed PF-SDPT algorithm compared to the PF-PT and the DFL both in terms of BER and MSE of the phase estimate.

\section{Appendix A - Posterior Cramér-Rao bound.}

Here, we wish to derive the PCRB associated to the phase distortion process $\left(\theta_{k}, \varepsilon_{k}\right)$ in order to obtain a lower bound for the MSE of the phase distortion estimates. Under the model (3), the Fisher information matrix (FIM) for $\left(\theta_{k}, \varepsilon_{k}\right)$ can be denoted by :

$$
J_{k}=\left(\begin{array}{ll}
J_{k}^{11} & J_{k}^{12} \\
J_{k}^{21} & J_{k}^{22}
\end{array}\right)
$$

that can be obtained by a recursive method which sequentially evaluates the inverse MSE of $\left(\theta_{k}, \varepsilon_{k}\right)$ as shown in [19] :

$$
\begin{aligned}
& J_{k+1}^{11}=H_{k}^{33}-\frac{\left(H_{k}^{13}\right)^{2}}{J_{k}^{11}+H_{k}^{11}} \\
& J_{k+1}^{12}=J_{k+1}^{21}=H_{k}^{23}-\frac{H_{k}^{13}\left(J_{k}^{12}+H_{k}^{12}\right)}{J_{k}^{11}+H_{k}^{11}} \\
& J_{k+1}^{22}=J_{k}^{22}+H_{k}^{22}-\frac{\left(J_{k}^{12}+H_{k}^{12}\right)^{2}}{J_{k}^{11}+H_{k}^{11}}
\end{aligned}
$$


Elements of matrix $H_{k}$ are obtained as follows :

$$
\begin{aligned}
H_{k}^{11} & =E\left[-\Delta_{\theta_{k}}^{\theta_{k}} \log p_{k}\right] \\
H_{k}^{12} & =E\left[-\Delta_{\theta_{k}}^{\varepsilon_{k}} \log p_{k}\right] \\
H_{k}^{13} & =E\left[-\Delta_{\theta_{k}}^{\theta_{k+1}} \log p_{k}\right] \\
H_{k}^{22} & =E\left[-\Delta_{\varepsilon_{k}}^{\varepsilon_{k}} \log p_{k}\right] \\
H_{k}^{23} & =E\left[-\Delta_{\varepsilon_{k}}^{\theta_{k+1}} \log p_{k}\right] \\
H_{k}^{33} & =E\left[-\Delta_{\theta_{k+1}}^{\theta_{k+1}} \log p_{k}\right]
\end{aligned}
$$

where

$$
p_{k}=p\left(\theta_{k+1} \mid \theta_{k}, \varepsilon_{k}\right) p\left(y_{k+1} \mid \theta_{k+1}, s_{\left\lfloor\frac{k+1}{\eta}\right\rfloor}\right)
$$

and, $\Delta$ denotes the second derivative operator, defined as $\Delta_{\varepsilon_{k}}^{\theta_{k+1}}=\partial^{2} / \partial \varepsilon_{k} \partial \theta_{k+1}$ and $\log ($.$) is the natural logarithm.$

Using equations (8) and (11), we obtain :

$$
\begin{aligned}
H_{k}^{11} & =1 / \sigma_{v}^{2} \\
H_{k}^{12} & =1 / \sigma_{v}^{2} \\
H_{k}^{13} & =-1 / \sigma_{v}^{2} \\
H_{k}^{22} & =1 / \sigma_{v}^{2} \\
H_{k}^{23} & =-1 / \sigma_{v}^{2} \\
H_{k}^{33} & =\frac{1}{\sigma_{v}^{2}}+\frac{E\left[2\left|s_{k+1}\right|^{2}\right]}{\sigma_{B}^{2}}=\frac{1}{\sigma_{v}^{2}}+\frac{2}{\sigma_{B}^{2}}
\end{aligned}
$$

Then, assuming that $J_{k+1}^{11}$ converges and using (17), (20), (22) and (25), we obtain for its limit the following result :

$$
J_{\infty}^{11}=\frac{2 \sigma_{v}^{2} \pm 2 \sqrt{\sigma_{v}^{2}\left(\sigma_{v}^{2}+2 \sigma_{B}^{2}\right)}}{2 \sigma_{v}^{2} \sigma_{B}^{2}}
$$

In the same way, $J_{\infty}^{12}=-1 / \sigma_{v}^{2}$ and $J_{\infty}^{22}$ diverges. Consequently, it is sufficient to assume that the parameter $\varepsilon_{k}$ is known. With this assumption, the Fisher information matrix $J_{k}$ corresponds to the $J_{k}^{11}$ and follows the recursion :

$$
J_{k+1}=\frac{1}{\sigma_{v}^{2}}+\frac{2}{\sigma_{B}^{2}}-\frac{1 / \sigma_{v}^{4}}{J_{k}+1 / \sigma_{v}^{2}}
$$

So the Cramér-Rao bound can be written recursively :

$$
C_{k+1}=\frac{1}{1 / \sigma_{v}^{2}+2 / \sigma_{B}^{2}-\frac{1 / \sigma_{v}^{4}}{1 / C_{k}+1 / \sigma_{v}^{2}}}
$$

Assuming convergence of the sequence, we deduce from (35) its asymptotic bound :

$$
C_{\infty}=-\frac{\sigma_{v}^{2}}{2}+\frac{\sqrt{\sigma_{v}^{2}\left(\sigma_{v}^{2}+2 \sigma_{B}^{2}\right)}}{2}
$$

\section{REFERENCES}

[1] T. Pollet, M. Van Bladel, and M. Moeneclaey, "BER sensitivity of OFDM systems to carrier frequency offset and wiener phase noise," IEEE Transactions on Communications, vol. 43, pp. 191-193, Feb. 1995.

[2] C. Garnier, L. Clavier, Y. Delignon, M. Loosvelt, and D. Boulinguez, "Multiple access for $60 \mathrm{GHz}$ mobile ad hoc network," in IEEE Vehicular Technology Conference, 2002, vol. 3, pp. 1517-1521.
[3] P.M. Djuric, J.H. Kotecha, J. Zhang, Y. Huang, T. Ghirmai, M.F. Bugallo, and J. Miguez, "Particle fi ltering," IEEE Signal Processing Magazine, vol. 5, pp. 19-38, Sep. 2003.

[4] T. Bertozzi, D. Le Ruyet, G. Rigal, and H. Vu-Thien, "Particle fi ltering for joint data-channel estimation in fast fading channels," in IEEE Vehicular Technology Conference, April 2003, vol. 4, pp. 2565-2569.

[5] E. Punskaya, C. Andrieu, A. Doucet, and W.J. Fitzgerald, "Particle fi ltering for multiuser detection in fading cdma channels," in Proc. 11th IEEE Workshop SSP, Singapore, 2001, pp. 38-41.

[6] P.O. Amblard, J.M. Brossier, and E. Moisan, "Phase tracking : what do we gain from optimality? Particle fi ltering versus phase-locked loops," Elsevier Signal Processing, vol. 83, pp. 151-167, Oct. 2003.

[7] Y. Huang and P.M. Djuric, "A hybrid importance function for particle fi ltering," IEEE Signal Processing Letters, vol. 11, no. 3, pp. 404406, Mar. 2004.

[8] E. Panayirci, H.A. Cirpan, and M. Moeneclaey, "A sequential monte carlo method for blind phase noise estimation and data detection," in 13th European Signal Processing Conference - EUSIPCO, Sept. 2005.

[9] M. Loosvelt, C. Garnier, and Y. Delignon, "Influence of pulse shaping on phase noise effects," in Proc. International Symposium on Image-Video Communications over fixed and mobile network (ISIVC), July 2004.

[10] Cong Ling, Kwok Hung Li, A.C. Kot, and Q.T. Zhang, "Multisampling decision-feedback linear prediction receivers for differential space-time modulation over rayleigh fast-fading channels," IEEE Transactions on Communications, vol. 51, no. 7, pp. 1214-1223, July 2003

[11] B.D. Hart and D.P. Taylor, "Extended MLSE receiver for the frequency-flat, fast-fading channel," IEEE Transactions on Vehicular Technology, vol. 46, no. 2, pp. 381-389, May 1997.

[12] B.D. Anderson and J.B. Moore, Optimal Filtering, Englewood Cliffs, NJ: Prentice-Hall, 1979.

[13] M.S. Arulampalam, S. Maskell, N. Gordon, and T. Clapp, "A tutorial on particles fi lters for online nonlinear/non-gaussian bayesian tracking," IEEE Trans. Signal Processing, vol. 50, pp. 174-188, Feb. 2002

[14] A. Doucet, N. De Freitas, and N. Gordon, Sequential Monte Carlo Methods in Practice, Springer-Verlag, 2001.

[15] M. Bolic, P.M. Djuric, and Sangjin Hong, "New resampling algorithms for particle fi lters," in Proc. IEEE ICASSP, April 2003.

[16] J.S. Liu and R. Chen, "Sequential monte carlo methods for dynamic systems," Journal of the American Statistical Association, vol. 93 no. 443, pp. 1032-1044, 1998.

[17] N. Gordon, D. Salmond, and A. Smith, "Novel approach to nonlinear/non-gaussian Bayesian state estimation," IEE Proceedings- $F$, vol. 140, pp. 107-113, April 1993.

[18] V. Zaritskii, V. Svetnik, and L. Shimelevich, "Monte carlo technique in problems of optimal data processing," Automation and remote control, pp. 95-103, 1975.

[19] P. Tichavsky, C.H. Muravchik, and A. Nehorai, "Posterior cramerrao bounds for discrete-time nonlinear fi ltering," IEEE Transactions on signal processing, vol. 46, no. 5, pp. 1386-1396, May 1998. 